\title{
Quantum Control of Light through an Atom-Molecule Dark State
}

\author{
Hui Jing ${ }^{1,2}$, Yuangang Deng ${ }^{1}$, and Weiping Zhang ${ }^{2}$ \\ ${ }^{1}$ Department of Physics, Henan Normal University, Xinxiang 453007, China \\ ${ }^{2}$ State Key Laboratory of Precision Spectroscopy, Department of Physics, \\ East China Normal University, Shanghai 200062, People's Republic of China
}

(Dated: November 2, 2018)

\begin{abstract}
We propose to use a quantized version of coherent two-color photoassociation to realize a hybrid device for quantum control of light. The dynamical features of this system are exhibited, including the slowing down or storage of light and the molecular matter-wave solitons. This may indicate a hybrid atom-molecule quantum device for storage and retrieve of optical information.
\end{abstract}

PACS numbers: 42.50.-p, 03.75.Pp, 03.70.+k

\section{INTRODUCTION}

The experimental realization of Bose-Einstein condensates (BECs) in ultracold atomic gases has led to tremendous advances from traditional atomic, molecular, and optical (AMO) physics [1] to current quantum information science [2, 3]. Recently, an intriguing atom-molecule dark state was observed in coherent two-color photoassociation (PA) [4], which has been considered as an efficient way to achieve higher production rates of molecules [5] from ultracold atoms. In view of their internal properties and long-range anisotropic interactions [6], the assembly of heteronuclear molecules $7-11]$ have also been actively pursued with various important applications 12 16], such as a polar molecular quantum computer [12, 17]. In the light of these developments it is timely to investigate the method of encoding and manipulating quantum optical state through the atom-molecule dark state. Such processes will provide new insights on current efforts of optical PA or quantum superchemistry with the goal of designing a hybrid atom-molecule device for quantum control of photonic information.

In this work we study such a scenario by transferring the quantum state of an associating light to an atomheternuclear molecule dark state [4, 5]. This allows us to study the effects of initial populations imbalance on the optical storage process. In particular, our work compares the results for atom-molecule systems with the more familiar light-storage schemes in atomic samples [18]. For a given number of atoms, the signal light is slowed more in the atom-molecule hybrid system, indicating some advantages over atomic slow-light media. Hence our present proposal, together with e.g. a cascaded molecular transition, may indicate a hybrid device for optical storage, processing, and retrieval.

\section{MODEL AND SOLUTIONS}

As Fig. 1 illustrated, the initial ultracold bosonic twospecies atomic condensates (with populations $N_{a}$ or $N_{b}$ ) are photoassociated into the excited molecular state $|e\rangle$ by a quantized signal light, which is then dumped into the ground molecular state $|g\rangle$ by another classical coupling

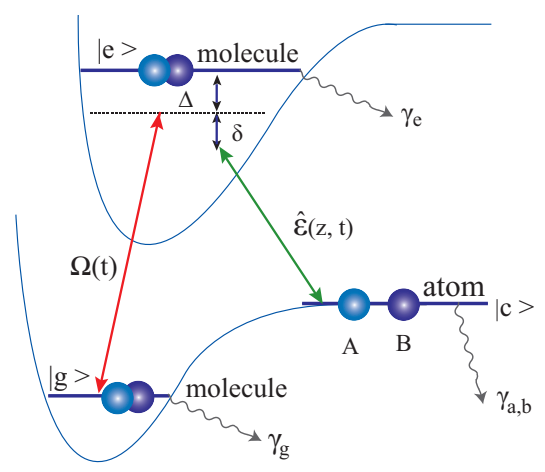

FIG. 1: (color online). Heteronuclear molecular creation in an initial two-species atomic Bose condensate via coherent twocolor PA, with free-quasi-bound-bound transition induced by a quantum signal light and a classical coupling field.

light. The signal pulse is described by the dimensionless operator

$$
\hat{E}(z, t)=\sqrt{\frac{\hbar \nu}{2 \epsilon_{0} L}} \hat{\mathcal{E}}(z, t) \exp \left(i \frac{\nu}{c}(z-c t)\right),
$$

where $L$ is the quantization length in the $z$ direction, $\nu$ is the PA light frequency and $\hat{\mathcal{E}}(z, t)$ is the slowly varying amplitude. We focus on the role of coherent couplings of photons and matter waves by ignoring the collisions of a dilute or Feshbach-resonance-tuned medium [19]. This is a safe approximation for the short lifetime of associated dimers [4]. The operators of signal light and matter waves satisfy the commutation relations, $\left[\hat{E}(z, t), \quad \hat{E}^{\dagger}\left(z^{\prime}, t\right)\right]=$ $\frac{\nu}{\epsilon_{0}} \delta\left(z-z^{\prime}\right),\left[\hat{\phi}_{i}(z, t), \hat{\phi}_{j}^{\dagger}\left(z^{\prime}, t\right)\right]=\delta_{i j} \delta\left(z-z^{\prime}\right)$, respectively. The dynamics of this system is described in the simplest level by the interaction Hamiltonian $(\hbar=1)$

$$
\begin{aligned}
\hat{\mathcal{H}}= & \Delta \int d z \hat{\phi}_{e}^{\dagger}(z, t) \hat{\phi}_{e}(z, t)+\delta \int d z \hat{\phi}_{a}^{\dagger}(z, t) \hat{\phi}_{a}(z, t) \\
& -\int d z\left[g \hat{\mathcal{E}}(z, t) \hat{\phi}_{e}^{\dagger}(z, t) \hat{\phi}_{a}(z, t) \hat{\phi}_{b}(z, t)\right. \\
& \left.+\Omega \hat{\phi}_{e}^{\dagger}(z, t) \hat{\phi}_{g}(z, t)+H . c .\right]
\end{aligned}
$$

where $\Delta$ or $\delta$ is the one- or two-photon detuning, $\Omega$ is the Rabi frequency of the coupling field, and $g=\wp \sqrt{\frac{\nu}{2 \hbar \epsilon_{0} V}}$ is 
the photon-matter waves coupling coefficient with $\wp$ being the transition-dipole moment of $|c\rangle-|e\rangle$ transition by $\hat{E}(z, t)$ [18]. Without loss of generality, we assume that the signal field amplitude $\hat{\mathcal{E}}$ and control field amplitude $\Omega$ are real whose phase factor can be absorbed by a global gauge transformation of the field operators [5]. Here we first drop off the usual kinetic and the trapping terms by considering a uniform system and the effects due to these terms will be discussed later.

With the slowly varying amplitude approximation [18], the propagation equation of the signal light can be written as

$$
\left(\frac{\partial}{\partial t}+c \frac{\partial}{\partial z}\right) \hat{\mathcal{E}}(z, t)=i g L \hat{\phi}_{a}^{\dagger}(z, t) \hat{\phi}_{b}^{\dagger}(z, t) \hat{\phi}_{e}(z, t) .
$$

Meanwhile, the evolutions of atomic field operators are described by the following Heisenberg equations

$$
\begin{aligned}
& \dot{\hat{\phi}}_{a}=-i \delta \hat{\phi}_{a}-\gamma_{a} \hat{\phi}_{a}+i g \hat{\mathcal{E}}^{\dagger} \hat{\phi}_{b}^{\dagger} \hat{\phi}_{e}, \\
& \dot{\hat{\phi}}_{b}=-\gamma_{b} \hat{\phi}_{b}+i g \hat{\mathcal{E}}^{\dagger} \hat{\phi}_{a}^{\dagger} \hat{\phi}_{e}, \\
& \dot{\hat{\phi}}_{e}=-i \Delta \hat{\phi}_{e}-\gamma_{e} \hat{\phi}_{e}+i g \hat{\mathcal{E}} \hat{\phi}_{a} \hat{\phi}_{b}+i \Omega \hat{\phi}_{g}, \\
& \dot{\hat{\phi}}_{g}=-\gamma_{g} \hat{\phi}_{g}+i \Omega \hat{\phi}_{e},
\end{aligned}
$$

where $\gamma_{a}, \gamma_{b}, \gamma_{e}$ and $\gamma_{g}$ denote the decay rates of corresponding matter-wave states. In order to obtain a closedform signal-light propagation equation, it is a key step to study the evolutions of the following hybrid operators,

$$
\begin{aligned}
& \frac{\partial}{\partial t}\left({\hat{\phi_{a}}}^{\dagger} \hat{\phi}_{b}^{\dagger} \hat{\phi}_{e}\right)=i g \hat{\mathcal{E}} \hat{\phi}_{a}^{\dagger} \hat{\phi}_{a} \hat{\phi}_{b}^{\dagger} \hat{\phi}_{b}+i \Omega \hat{\phi}_{a}^{\dagger} \hat{\phi}_{b}^{\dagger} \hat{\phi}_{g} \\
& -\left(\gamma_{2}-i \Delta-i \delta\right) \hat{\phi}_{a}^{\dagger} \hat{\phi}_{b}^{\dagger} \hat{\phi}_{e} \\
& -i g \hat{\mathcal{E}}\left(\hat{\phi}_{a}^{\dagger} \hat{\phi}_{a}+\hat{\phi}_{b}^{\dagger} \hat{\phi}_{b}\right) \hat{\phi}_{e}^{\dagger} \hat{\phi}_{e} \\
& \begin{aligned}
\frac{\partial}{\partial t}\left(\hat{\phi}_{a}^{\dagger} \hat{\phi}_{b}^{\dagger} \hat{\phi}_{g}\right)= & -\left(\gamma_{1}-i \delta\right) \hat{\phi}_{a}^{\dagger} \hat{\phi}_{b}^{\dagger} \hat{\phi}_{g}+i \Omega \hat{\phi}_{a}^{\dagger} \hat{\phi}_{b}^{\dagger} \hat{\phi}_{e} \\
& -i g \hat{\mathcal{E}}\left(\hat{\phi}_{a}^{\dagger} \hat{\phi}_{a}+\hat{\phi}_{b}^{\dagger} \hat{\phi}_{b}\right) \hat{\phi}_{e}^{\dagger} \hat{\phi}_{g}
\end{aligned}
\end{aligned}
$$

with the transversal decay rates $\gamma_{1}=\gamma_{a}+\gamma_{b}+\gamma_{g}$ and $\gamma_{2}=\gamma_{a}+\gamma_{b}+\gamma_{e}$. These equations can be rewritten as

$$
\begin{aligned}
\hat{\phi}_{a}^{\dagger} \hat{\phi}_{b}^{\dagger} \hat{\phi}_{e}= & -\frac{i}{\Omega} \frac{\partial}{\partial t}\left(\hat{\phi}_{a}^{\dagger} \hat{\phi}_{b}^{\dagger} \hat{\phi}_{g}\right)-\frac{i}{\Omega}\left(\gamma_{1}-i \delta\right) \hat{\phi}_{a}^{\dagger} \hat{\phi}_{b}^{\dagger} \hat{\phi}_{g} \\
+ & \frac{g}{\Omega} \hat{\mathcal{E}}\left(\hat{\phi}_{a}^{\dagger} \hat{\phi}_{a}+\hat{\phi}_{b}^{\dagger} \hat{\phi}_{b}\right) \hat{\phi}_{e}^{\dagger} \hat{\phi}_{g} \\
\hat{\phi}_{a}^{\dagger} \hat{\phi}_{b}^{\dagger} \hat{\phi}_{g}= & -\frac{i}{\Omega} \frac{\partial}{\partial t}\left(\hat{\phi}_{a}^{\dagger} \hat{\phi}_{b}^{\dagger} \hat{\phi}_{e}\right)-\frac{g}{\Omega} \hat{\mathcal{E}}_{\phi_{a}^{\dagger}}^{\dagger} \hat{\phi}_{a} \hat{\phi}_{b}^{\dagger} \hat{\phi}_{b} \\
& -\frac{i}{\Omega}\left(\gamma_{2}-i \Delta-i \delta\right) \hat{\phi}_{a}^{\dagger} \hat{\phi}_{b}^{\dagger} \hat{\phi}_{e} \\
& +\frac{g}{\Omega} \hat{\mathcal{E}}\left(\hat{\phi}_{a}^{\dagger} \hat{\phi}_{a}+\hat{\phi}_{b}^{\dagger} \hat{\phi}_{b}\right) \hat{\phi}_{e}^{\dagger} \hat{\phi}_{e}
\end{aligned}
$$

It should be noted that Eq. (77) and Eq. (8) can be greatly simplified under the weak excitation approximation (WEA): the control field is much stronger than the signal light at all times and thus the density of signal photons can be taken as much less than that of atoms. This means that only a small ratio of atoms are converted into molecules, which is the case in the recent two-color PA experiment [4]. With the WEA at hand, after some algebra we find in the lowest non-vanishing order

$$
\hat{\phi}_{a}^{\dagger} \hat{\phi}_{b}^{\dagger} \hat{\phi}_{g} \approx-\frac{g \hat{\mathcal{E}}}{\Omega} \hat{\phi}_{a}^{\dagger} \hat{\phi}_{a} \hat{\phi}_{b}^{\dagger} \hat{\phi}_{b} \text {. }
$$

Hence Eq. (7) can be rewritten as

$$
\hat{\phi}_{a}^{\dagger} \hat{\phi}_{b}^{\dagger} \hat{\phi}_{e} \approx i \frac{g N_{a} N_{b}}{\Omega^{2}} \frac{\partial}{\partial t} \hat{\mathcal{E}}(z, t)-i \frac{g N_{a} N_{b}}{\Omega^{3}} \frac{\partial \Omega(t)}{\partial t} \hat{\mathcal{E}}(z, t),
$$

where $N_{a, b}=\hat{\phi}_{a, b}^{\dagger} \hat{\phi}_{a, b}$ is the population of atoms $\mathrm{A}$ or B, which can be assumed as constant in the WEA. Substituting Eq. (10) into Eq. (3) yields

$$
\begin{aligned}
& \left(\frac{\partial}{\partial t}+\frac{c}{1+\frac{g^{2} L N_{a} N_{b}}{\Omega^{2}}} \frac{\partial}{\partial z}\right) \hat{\mathcal{E}}(z, t)= \\
& \quad=\frac{1}{1+\frac{g^{2} L N_{a} N_{b}}{\Omega^{2}}} \frac{g^{2} L N_{a} N_{b}}{\Omega^{3}} \frac{\partial \Omega}{\partial t} \hat{\mathcal{E}}(z, t) .
\end{aligned}
$$

Clearly, for a time-independent coupling field, we have a steady group velocity of the signal, and the temporal profile or the spectrum of the signal pulse remains unchanged during its slowing down process, just as in a three-level atomic ensemble [18]. For a time-dependent coupling field, however, the rand-hand side of Eq. (11) leads to an adiabatic Raman enhancement of the signal pulse

$$
\hat{\mathcal{E}}(z, t)=\frac{\cos \theta(t)}{\cos \theta(0)} \hat{\mathcal{E}}\left(z-\int_{0}^{t} v_{g} d t^{\prime}, 0\right),
$$

where $v_{g}=c \cos ^{2} \theta$ is the group velocity of the signal light and $\theta$ is the mixing angle between light and matter-wave components, i.e.,

$$
\begin{aligned}
\tan ^{2} \theta & =g^{2} N_{a} N_{b} L / \Omega^{2}(t), \\
v_{g}^{-1} & =\left(1+\frac{\tilde{g}^{2} N_{a} N_{b}}{\Omega^{2}}\right) / c,
\end{aligned}
$$

with $\tilde{g}=g \sqrt{L}$. Obviously, if the classical field is adiabatically turned off by rotating the mixing angle $\theta$ for $\pi / 2$, the signal light will be fully stopped within the medium or in the created atom-molecule dark state [4].

For the atomic slow-light medium [18], the group velocity of signal light is: $v_{g}=c /\left(1+\frac{g^{2} N}{\Omega^{2}}\right)$, i.e., being proportional to $\sim N^{-1}$, where $N$ can be regarded as the number of initial trapped atoms in the WEA; however, in our situation, this velocity is proportional to $\sim N^{-2}$ (for an initial balanced sample $N_{a}=N_{b}=N / 2$ ). Hence the technique of atom-molecule dark state may have some advantage over the scheme of purely atomic spin waves since the signal light can be much slowed down by starting from the same total number of atoms. 
The quantum state transfer process is also observed through the form of the closed-channel molecular field. From Eqs. (7-9), it is straightforward to find

$$
\begin{array}{r}
\hat{\phi}_{g}(z, t) \approx k \hat{\mathcal{E}}\left(z-\int_{0}^{t} v_{g} d t^{\prime}, 0\right), \\
k=-\frac{g \sqrt{N_{a} N_{b}}}{\Omega(0)} \sqrt{\frac{\Omega^{2}(0)+\tilde{g}^{2} N_{a} N_{b}}{\Omega^{2}(t)+\tilde{g}^{2} N_{a} N_{b}}},
\end{array}
$$

Obviously, for the initial stage $\left(\Omega^{2} \gg \tilde{g}^{2} N_{a} N_{b}\right)$, we have a purely photonic state, i.e., $\theta(0)=0$ or

$$
\hat{\phi}_{g}(z, 0)=0
$$

but when the coupling light is shut down adiabatically, the quantum state of the associating light is fully encoded into the created molecules via the mapping

$$
\sqrt{L} \hat{\phi}_{g}(z, t)=-\hat{\mathcal{E}}\left(z-\int_{0}^{t} v_{g} d t^{\prime}, 0\right),
$$

which thus indicates a possible quantum memory device based on coherent two-color PA technique.

\section{ROLE OF POPULATIONS IMBALANCE}

As Fig. 2 shows, the initial populations imbalance of the atoms A, B $\left(\eta=N_{b} / N_{a}\right)$ also plays a role in the optical storage process, and the optimal conversion is reached for the balanced case $(\eta=1)$. In our calculations, the initial total atomic number is taken as $N=N_{a}+N_{b}=3.0 \times 10^{6}$. In current experimental conditions [20], the strength of coupling field can be chosen as (in the unit of $\mathrm{MHz}$ )

$$
\begin{aligned}
\Omega(t)= & 10 \pi(1-0.5 \tanh [0.15(t-15)] \\
& +0.5 \tanh [0.15(t-125)]),
\end{aligned}
$$

which guarantees that the group velocity can be adiabatically reduced to zero in the photon-storage stage and then the signal light can be re-accelerated in the retrieval stage. The control field being similar to Eq. (16) is also used in the atomic slow-light medium [18]. For the other cases of initial population imbalance, e.g., $\eta=15$ as in the very recent experiment of creating polar molecules $\mathrm{KRb}$ [7], some deviation from the optimized case can appear (see Fig. 2).

Fig. 3 shows the different features of slow light propagation in four different kinds of matter-wave mediums: (i) the atomic ensemble, (ii)-(iii) the assembly of homonuclear or heteronuclear diatomic molecules [5, 21], and (iv) the assembly of heteronuclear triatomic molecules $\mathrm{ABC}$ [5] (for the trimer case, the calculation of the group velocity is completely similar to the above dimer cases). In addition, we see from Fig. 3 that, by choosing a higher initial atomic populations, the optical storage process can be significantly improved.

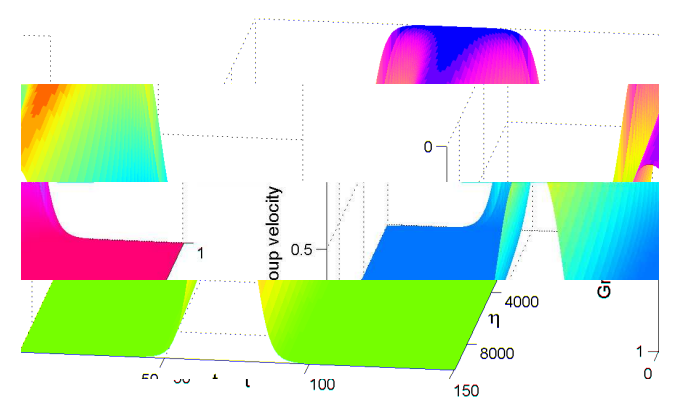

FIG. 2: (color online). The group velocity of signal light as a function of time for different populations imbalances $\eta$. The quantized length $L=1 \mathrm{~mm}$, the coupling coefficient $g=10$, the velocity is scaled by $c$ and the time is in the unit of $\mu$ s.

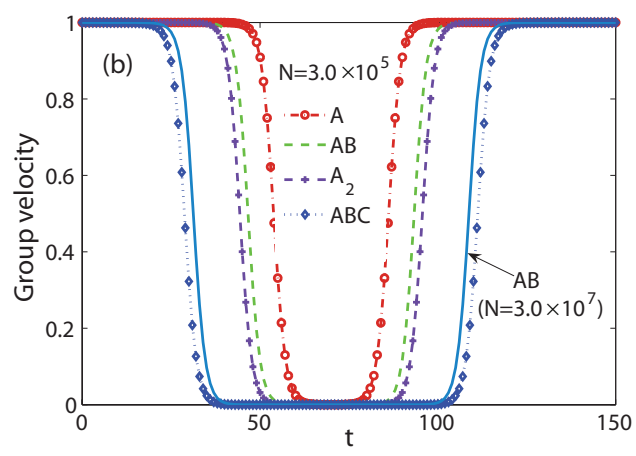

FIG. 3: (color online). The group velocity of the signal light as a function of time in different matter-wave mediums, with the optimal or balanced initial populations imbalance.

It is worth mentioning that in the above discussions we have ignored the decay of molecular states. However, it is readily to show that, after including these decay terms, the group velocity of the signal light is still in the form of Eq. (13) but with the following substitution

$$
\Omega \rightarrow \sqrt{\Omega^{2}+\gamma_{1} \gamma_{2}} .
$$

Clearly, due to the decay terms, one may reach a nonzero group velocity even when the classical field is turned off. For the typical parameters of the molecules KRb 20], we can take $N_{\mathrm{K}}=1.0 \times 10^{6}, N_{\mathrm{Rb}}=5.0 \times 10^{6}, \tilde{g} \approx 50 \mathrm{~s}^{-1}$, and

$$
\gamma_{1}=2 \pi \times 97 \mathrm{~Hz}, \gamma_{2}=2 \pi \times 5.7 \mathrm{MHz} .
$$

The velocity limit can be estimated as $v_{g}^{c} \approx 0.524 \mathrm{~km} \cdot \mathrm{s}^{-1}$. In particular, for a sufficient state transfer, the PA time duration should satisfy $t \ll \gamma_{1}^{-1} \sim 1.6 \mathrm{~ms}$ [22, 23], which can be fulfilled in current experiments [24, 25].

We also note that some optimized methods exist for a maximum efficiency of optical storage and retrieval, such as the recent works of Novikova et al. by using an optimized signal pulse shape in an atomic medium [26, 27]. For the present atom-molecule system, in order to avoid 
incoherent absorptive loss, the frequency components of the signal pulse must fit well with the slow-light spectral window, i.e., $t_{s}^{-1} \ll \sqrt{d} v_{g} / L \sim N^{-1}$, where $t_{s}$ is the temporal length of the signal pulse, $d=g^{2} N_{a} N_{b} L /(\gamma c)$ is optical depth of the medium. Thereby, to avoid "leakage" of the pulse outside the medium, the following condition should be fulfilled

$$
v_{g} t_{s} \sim N^{-1} \ll L .
$$

which means that $v_{g}$ must be small enough for the entire signal pulse to be spatially compressed into the medium, with also a large optical depth d. For contrast, by using a purely atomic medium, we have $d=g^{2} N L /(\gamma c)$ 26] and thus $v_{g} t_{s} \sim N^{-1 / 2}$ [27]. Obviously, for the same initial total atomic number, the quantum light storage using the atom-molecule dark state may have some advantage over the familiar atomic spin-wave scheme.

\section{MOLECULAR SOLITON LASER}

Now we show that, by taking into account of the particle collisions in the quantum state transfer process, it is also possible to realize a molecular matter-wave soliton laser. To this end, we consider coherent atom-molecule conversion process which is described by the following total Hamiltonian

$$
\hat{H}_{\text {tot }}=\hat{H}_{0}+\hat{H}_{\text {coll }}+\hat{\mathcal{H}}
$$

where $\hat{\mathcal{H}}$, as in Eq. (2), denotes the coherent free-quasibound-bound transition, $\hat{H}_{0}$ and $\hat{H}_{\text {coll }}$ describe the free motions and the particle collisions, respectively,

$$
\begin{aligned}
& H_{0}=\sum_{i} \int d z \hat{\phi}_{i}^{\dagger}\left(-\frac{1}{2 m_{i}} \frac{\partial^{2}}{\partial z^{2}}+V_{i}\right) \hat{\phi}_{i}, \\
& H_{\text {coll }}=\sum_{i, j} U_{i j} \int d z \hat{\phi}_{i}^{\dagger} \hat{\phi}_{j}^{\dagger} \hat{\phi}_{j} \hat{\phi}_{i},
\end{aligned}
$$

were $V_{i}(z)(i, j=a, b, g)$ denotes the longitudinal external effective potential and one can choose $V_{a}(z)=V_{b}(z)=0$ in the following derivation [18], $m_{i}$ is the particle mass, $U_{i j}$ denotes the $s$-wave scattering collisions between the particles [28]. Then the evolution of molecular matterwave field is written as

$$
\begin{aligned}
i \frac{\partial \hat{\phi}_{g}}{\partial t} & =\left[-\frac{1}{2\left(m_{a}+m_{b}\right)} \frac{\partial^{2}}{\partial z^{2}}+U_{a b} \hat{\phi}_{a}^{\dagger} \hat{\phi}_{b}\right. \\
& \left.+U_{g g} \hat{\phi}_{g}^{\dagger} \hat{\phi}_{g}+V_{g}(z)\right] \hat{\phi}_{g}-\Omega \hat{\phi}_{e} .
\end{aligned}
$$

For simplicity, we consider an initially trapped atomic ensemble and also introduce the mean-field approach by replacing the operators by the $c$-numbers, i.e., $\hat{\phi}_{i}(z, t) \rightarrow$ $\Phi_{i}(z, t)$. Thereby, for the closed-channel molecules, i.e., with the mixing angle $\theta=\pi / 2$, we obtain the nonlinear mean-field Gross-Pitaevskii equation

$$
i \frac{\partial \Phi_{g}}{\partial t}=-\frac{1}{2\left(m_{a}+m_{b}\right)} \frac{\partial^{2} \Phi_{g}}{\partial z^{2}}+V_{e f f} \Phi_{g}+U_{g g}\left|\Phi_{g}^{0}\right|^{2} \Phi_{g},
$$

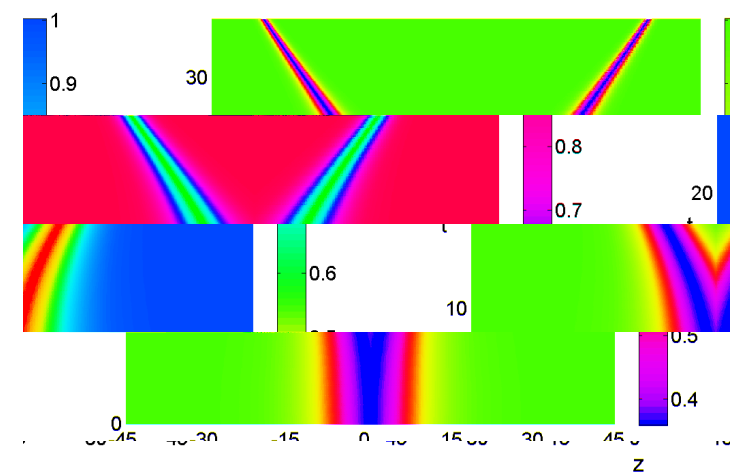

FIG. 4: (color online). Splitting grey solitons under the influence of background amplitude decreasing, for $q=0.8$. The units of time and trap size are in $\mu$ s and $\mu \mathrm{m}$, respectively.

where the effective potential $V_{\text {eff }}=V_{g}+\sqrt{N_{a} N_{b}} U_{a b}$ can also be moved by suitably tuning the value of $V_{g}$ [18]. For $a_{g g}>0$, due to some balance between the repulsive molecular collisions and the molecular kinetic energy, the above well-known nonlinear equation can support a graysoliton solution 29, 30]

$$
\Phi_{g}=\Phi_{g}^{0}(z, t)\left\{i \sqrt{1-q^{2}}+q \tanh \left[\frac{q}{\sqrt{\alpha}}\left(z-z_{0}(t)\right)\right]\right\},
$$

with $\alpha=\left(\sqrt{4 \pi a_{g g}}\left|\Phi_{g}^{0}\right|\right)^{-1} \cdot V_{g}$ is chosen such that $V_{\text {eff }}=$ 0 , the slowly-varying background function is

$$
\Phi_{g}^{0}(z, t)=\left\langle\hat{\phi}_{g}\right\rangle \exp \left(-i \int_{t_{0}}^{t} U_{g g}\left|\left\langle\hat{\phi}_{g}\right\rangle\right|^{2} d t^{\prime}\right)
$$

and $z$ is the inside-trap position. In addition, the "grayness" parameter is

$$
q=\sqrt{1-\left(v_{\nu} / v_{s}\right)^{2}} \leq 1,
$$

with the Bogoliubov sound speed 31]

$$
v_{s}=\left[U_{g g}\left|\Phi_{g}^{0}\right|^{2} /\left(m_{a}+m_{b}\right)\right]^{1 / 2},
$$

and the dark-soliton speed $v_{\nu}=\dot{\mathrm{z}}_{0}(t), z_{0}(t)$ being the central position of the molecular matter-wave soliton. Clearly, $q=1$ corresponds to a dark soliton with $100 \%$ density depletion. We see from Fig. 4 that, by applying two counter-propagating control fields, a second-order molecular grey soliton $(q=0.8)$ starting from the same position $(z=0)$ can split into two solitons propagating at opposite directions.

\section{CONCLUSION}

In conclusion, we have studied the slow light and quantum optical storage process in coherent two-color PA process by considering a quantized associating light. By taking into account of the particle collisions, one may also 
create the molecular matter-wave solitons. This may indicate a hybrid atom-molecule quantum device for storage and retrieve of optical information. It is straightforward to study other interesting configurations, such as the light-molecule entanglement by applying a nonclassical PA light [19, 32], or the quantum switch by considering a multi-level atom-molecule system.

As far as we know, our work sets up the first link between the research fields of quantum memory and coherent atom-molecule conversion. Due to the rapid experimental advances in both two fields, this atom-molecule system may be potentially useful for designing a hybrid atom-molecule quantum device of optical storage, processing, and retrieval. In the future, we plan to study the quantum memory in a boson-fermion mixture [5, 8], the slow light in the BEC-BCS crossover of a fermionic atomic sample [10], and the polarization rotation of slow light [33] with the Laguerre-Gaussian signal modes.

\section{Acknowledgments}

H.J. and Y.D. are supported by the National Science Foundation of China (Grant No. 10874041), the NCET, and the ECNU Key Lab Open Fund. Weiping Zhang is supported by the National Science Foundation of China (Grant No. 10588402 and No. 10474055), the National Basic Research Program of China (Grant No. 2006CB921104), the Science and Technology Commission of Shanghai Municipality (Grant No. 06JC14026 and No. 05PJ14038), the Program of Shanghai Subject Chief Scientist (Grant No. 08XD14017), the Program for Changjiang Scholars and Innovative Research Team, Shanghai Leading Academic Discipline Project (Grant No. B480), and the Research Fund for the Doctoral Program of Higher Education (Grant No. 20040003101).
[1] P. Meystre, Atom Optics (Springer-Verlag, Berlin, 2001).

[2] A. Ekert, and R. Josza, Rev. Mod. Phys. 68, 733 (1996).

[3] A. Furusawa, J. L. Sørensen, S. L. Braunstein, C. A. Fuchs, H. J. Kimble, and E. S. Polzik, Science 282, 706 (1998).

[4] K. Winkler, G. Thalhammer, M. Theis, H. Ritsch, R. Grimm, and J. H. Denschlag, Phys. Rev. Lett. 95, 063202 (2005); M. Mackie, R. Kowalski, and J. Javanainen, ibid. 84, 3803 (2000).

[5] H. Y. Ling, H. Pu, and B. Seaman, Phys. Rev. Lett. 93, 250403 (2004); A.Nunnenkamp, D. Meiser, and P. Meystre, New J. Phys. 8, 88 (2006); H. Jing, Y. J. Jiang, Weiping Zhang, and P. Meystre, ibid. 10, 123005 (2008).

[6] L. Santos, G. V. Shlyapnikov, P. Zoller, and M. Lewenstein, Phys. Rev. Lett. 85, 1791 (2000).

[7] M. W. Mancini, G. D. Telles, A. R. L. Caires, V. S. Bagnato, and L. G. Marcassa, Phys. Rev. Lett. 92, 133203 (2004).

[8] A. G. Truscott, K. E. Strecker, W. I. McAlexander, G. B. Partridge, and R. G. Hulet, Science 291, 2570 (2001).

[9] J. M. Sage, S. Sainis, T. Bergeman, and D. DeMille, Phys. Rev. Lett. 94, 203001 (2005).

[10] M. Taglieber, A. C. Voigt, T. Aoki, T. W. Hänsch, and K. Dieckmann, Phys. Rev. Lett. 100, 010401 (2008).

[11] J. Deiglmayr, A. Grochola, M. Repp, K. Mörtlbauer, C. Glück, J. Lange, O. Dulieu, R. Wester, and M. Weidemüller, Phys. Rev. Lett. 101, 133004 (2008).

[12] D. DeMille, Phys. Rev. Lett. 88, 067901 (2002), S. F. Yelin, K. Kirby, R. Côté, Phys. Rev. A 74, 050301(R) (2006).

[13] A. Micheli, G. K. Brennen, and P. Zoller, Nat. Phys. 2, 341 (2006).

[14] P. Rabl, D. DeMille, J. M. Doyle, M. D. Lukin, R. J. Schoelkopf, and P. Zoller, Phys. Rev. Lett. 97, 033003 (2006).

[15] E. R. Hudson, H. J. Lewandowski, B. C. Sawyer, and J. Ye, Phys. Rev. Lett. 96, 143004 (2006).

[16] J. J. Hudson, B. E. Sauer, M. R. Tarbutt, and E. A. Hinds, Phys. Rev. Lett. 89, 023003 (2002).

[17] K. K. Ni, S. Ospelkaus, M. H. G. de Miranda, A. Péer, B.
Neyenhuis, J. J. Zirbel, S. Kotochigova, P. S. Julienne, D. S. Jin, and J. Ye, Science 322, 231 (2008).

[18] M. Fleischhauer, and M. D. Lukin, Phys. Rev. Lett. 84, 5094 (2000); M. Fleischhauer, and M. D. Lukin, Phys. Rev. A 65, 022314 (2002).

[19] S. A. Haine, M. K. Olsen, and J. J. Hope, Phys. Rev. Lett. 96, 133601 (2006).

[20] S. Ospelkaus, A.Pe'er, K. K. Ni, J. J. Zirbel, B. Neyenhuis, S. Kotochigova, P. S. Julienne, J. Ye, and D. S. Jin, Nat. Phys. 4, 622 (2008).

[21] H. Jing and M.-S. Zhan, Eur. Phys. J. D 42, 183 (2007); X.-T. Zhou, X.-J. Liu, H. Jing, C. H. Lai, and C. H. Oh, arXiv: quant-ph/0701080 (2007).

[22] S. Chen, Y. A. Chen, T. Strassel, Z. S. Yuan, B. Zhao, J. Schmiedmayer, and J. W. Pan, Phys. Rev. Lett. 97, 173004 (2006).

[23] J. Simon, H. Tanji, J. K. Thompson, and V. Vuletić, Phys. Rev. Lett. 98, 183601 (2007).

[24] R. Zhao, Y. O. Dudin, S. D. Jenkins, C. J. Campbell, D. N. Matsukevich, T. A. B. Kennedy, and A.Kuzmich, Nature Phys. 5, 100 (2009); B. Zhao, Y. A. Chen, X. H. Bao, T. Strassel, C. S. Chuu, X. M. Jin, J. Schmiedmayer, Z. S. Yuan, S. Chen, and J. W. Pan, Nature Phys. 5, 95 (2009).

[25] N. S. Ginsberg, S. R. Garner, and L. V. Hau, Nature (London) 445, 623 (2007).

[26] A. V. Gorshkov, A. André, M. Fleischhauer, A. S. Sørensen, and M. D. Lukin, Phys. Rev. Lett. 98, 123601 (2007).

[27] I. Novikova, A. V. Gorshkov, D. F. Phillips, A. S. Sørensen, M. D. Lukin, and R. L. Walsworth, Phys. Rev. Lett. 98, 243602 (2007).

[28] M. Mackie, K. Härkönen, A. Collin, K. A. Suominen, and J. Javanainen, Phys. Rev. A 70, 013614 (2004).

[29] T. Busch, and J. R. Anglin, Phys. Rev. Lett. 84, 2298 (2000).

[30] J. Denschlag, J. E. Simsarian, D. L. Feder, C. W. Clark, L. A. Collins, J. Cubizolles, L. Deng, E. W. Hagley, K. Helmerson, W. P. Reinhardt, S. L. Rolston, B. I. Schneider, and W. D. Phillips, Science, 287, 97 (2000). 
[31] M. R. Andrews, D. M. Kurn, H. J. Miesner, D. S. Durfee, C. G. Townsend, S. Inouye, and W. Ketterle, Phys. Rev. Lett. 79, 553 (1997).

[32] H. Jing, J.-L. Chen, and M.-L. Ge, Phys. Rev. A 63, 015601 (2000); S. A. Haine and J. J. Hope, ibid. 72,
033601 (2005).

[33] J. Ruseckas, G. Juzeliūnas, P. Öberg, and S. M. Barnett, Phys. Rev. A 76, 053822 (2007). 